\title{
Natural killer cells go inside: Entosis versus cannibalism
}

\author{
Youcun Qian ${ }^{1}$, Yufang Shi ${ }^{1,2}$ \\ ${ }^{1}$ Key Laboratory of Stem Cell Biology, Institute of Health Sciences, Shanghai Institutes for Biological Sciences, Chinese Academy of \\ Sciences, \& Shanghai Jiaotong University School of Medicine, 225 South Chongqing Road, Shanghai 200025, China; ${ }^{2}$ Department \\ of Molecular Genetics, Microbiology and Immunology, UMDNJ-Robert Wood Johnson Medical School, 661 Hoes Lane, Piscataway, \\ NJ 08854, USA \\ Cell Research (2009) 19:1320-1321. doi: 10.1038/cr.2009.134; published online 3 December 2009
}

The cell-in-cell phenomenon is frequently observed in tumor samples, especially in those highly malignant or metastatic tumors. It is believed that through this mechanism, cancer cells could ingest immune cells such as neutrophils and lymphocytes, which otherwise kill tumor cells. Tumor cells could also phagocytose tumor cells-a phenomenon resembles autophagic digestion of cellular organelles under starvation. This cell-eat-cell phenomenon is called cannibalism [1-2]. Therefore, tumors may take advantage of cell cannibalism for survival and immune evasion. It was recently discovered that the cell-in-cell phenomenon also happens in epithelial cells and is referred to as entosis [3]. Entosis is a homogeneous cell-in-cell phenomenon while cannibalism can be either homogeneous or heterogeneous cell-in-cell structures [4-5]. The process of entosis is different from cannibalism in that entosis is a live cell invasion while cannibalism has no selectivity for dead cells or live cells. Interestingly, the studies by Wang et al. [6] presented in this issue revealed that internalization of natural killer (NK) cells in tumor cells is a distinct type of cell-in-cell phenomenon that is different from cannibalism and entosis.

The phenomenon of internalization

Correspondence: Yufang Shi

E-mail: shiyu@umdnj.edu of NK cells to tumor cells was reported more than two decades ago [7]. However, the mechanisms of the process and the fate of the internalized NK cells have yet to be investigated. Here, Wang et al. reported that internalization of NK cells is an active process and only involves live cells, which is similar to the process of entosis, but different from cannibalism. In entosis, live epithelial cells or tumor cells detach from extracellular metrix (ECM) and then invade their neighbor cells. This process is also different from normal phagocytosis of apoptotic cells. Entosis depends on conjugations or adherens junctions and needs Rho and ROCK activities for internalization, suggesting that entosis is an active process and requires actin polymerization. Similarly, Wang et al. found that the internalization of NK cells also involves actin polarization and is an active process. Only live NK cells enter into neighboring tumor cells. Transmission electron microscopic analyses revealed that the NK cell plasma membrane remains connected to its target membrane, suggesting the involvement of adherens junction. It is well known that E-cadherin is involved in cell-cell adhesion and compaction [8]. Indeed, knockdown of E-cadherin using siRNA severely impaired cell-cell contact and internalization of NK cells to A431 tumor cells. Since cadherin proteins require calcium for function, Wang et al. also found that calcium chelating reagent EGTA inhibits cell-cell conjugation or junctions and consequently NK cells internalization to tumor cells. Ezrin, an actin-binding protein, plays an important role in the regulation of actin and cytoskeleton functions [9]. It was also shown to be involved in membrane-cytoskeleton dynamics and in the formation of immunological synapse between NK cells and target tumor cells. When ezrin was knocked down, Wang et al. found that internalization of NK cells to A431 target cells was completely inhibited, However, ezrin knockdown did not affect membrane association of NK cells and target cells, suggesting ezrin functions in internalization after cell conjugation/ junction. As ezrin activity is shown to be functionally regulated by site-specific phosphorylation [10], the authors further investigated the function of ezrin's phosphorylation by PKA and found that PKA inhibitor prohibited NK cell internalization, confirming an essential role of ezrin in NK cells internalization to tumor cells. Although the process of NK cells internalization to tumor cells is similar to entosis, the fate and death mechanisms of the internalized NK cells seem to be quite different. In entosis, internalized homogenous cells go through non-apoptotic lysosomemediated degradation. However, Wang et al. found that some NK cells in target tumor cells go through typical apoptotic cell death. Activation of caspase 3 was 
observed in internalized NK cells but not in target A431 cells. TUNEL assay also showed DNA fragmentation specifically in internalized NK cells. Furthermore, caspase inhibitors attenuated the apoptotic process. Therefore, this study strongly suggests that NK cell internalization is a distinct type of cell-in-cell phenomena.

Tumor cells take advantage of cannibalism to kill immune cells such as neutrophils and lymphocytes for survival and immune evasion. Entosis is a homogenous cell-eat-cell phenomenon which happens in both normal and tumor cells. Its physiological functions are yet to be identified, although entosis was shown to have tumor suppressive effect in soft agar assay. NK cells internalization to tumor cells is a heterozygous cell-in-cell phenomenon also called emperpolesis, which is expected to be similar to cannibalism. Unexpectedly, mechanistic investigation by Wang et al. showed that NK cells internalization needs cell conjugation and actin cytoskeleton reorganization in target cells in order to allow NK cells entry, which also happens during the entosis process. It is important to emphasize that the same group previously found that NK cell internalization led to target tumor cell death and NK cell self-destruction, suggesting that NK cells may use this kind of cell-in-cell pathway to kill target tumor cells in addition to the usual target cell killing through the cytolytic effect. This potential tumor suppressive function is similar to that in entosis. However, it is still possible that target tumor cells may use NK cells internalization for survival and escape from immunesurveillance as in cannibalism. The exact physiological and pathological functions of NK cells internalization to tumor cells need to be further investigated. The current study leads the way of dissecting the mechanism of NK cell internalization process, opening a new branch for NK cell biology and tumor immunology in this fascinating cell-eatcell research field.

\section{References}

1 Stefano Fais. Cannibalism: A way to feed on metastatic tumors. Cancer Lett 2007; 258:155-164.

2 Luana Lugini, Paola Matarrese, Tinari A, et al. Cannibalism of live lymphocytes by human metastatic but not primary melanoma cells. Cancer Res
2006; 66:3629-3638.

3 Overholtzer M, Mailleux AA, Mouneimne $\mathrm{G}$, et al. A nonapoptotic cell death process, entosis, that occurs by cell-incell invasion. Cell 2007; 131:966-979.

4 White E. Entosis: It's a cell-eat-cell world. Cell 2007; 131:840-842.

5 Xia P, Wang S, Guo Z, et al. Emperipolesis, entosis and beyond: Dance with fate. Cell Res 2008; 18:705-707.

6 Wang S, Guo Z, Xia P, et al. Internalization of NK cells into tumor cells requires ezrin and leads to a programmed cell-in-cell death. Cell Res 2009; 19:1350-1362.

7 Wang X, Li W. Mechanisms of natural killer cell-mediated tumor cell cytolysis at a single cell level. $J$ Med Col PLA 1987; 2:107-117.

8 Adams CL, Chen YT, Smith SJ, Nelson WJ. Mechanisms of epithelial cell-cell adhesion and cell compaction revealed by high-resolution tracking of E-cadherin-green fluorescent protein. J Cell Biol 1998; 142:1105-1119.

9 Yao X, Cheng L, Forte JG. Characterization of the interaction of gastric ezrin with actin. J Biol Chem 1996; 271:72247229.

10 Zhou R, Cao X, Watson C, et al. Characterization of PKA-mediated phosphorylation of ezrin in gastric parietal cell activation. J Biol Chem 2003; 278:35651-35659. 